\title{
Weakly mixing rank-one transformations conjugate to their squares
}

by

\author{
Alexandre I. Danilenko (Bonn and Kharkov)
}

\begin{abstract}
Utilizing the cut-and-stack techniques we construct explicitly a weakly mixing rigid rank-one transformation $T$ which is conjugate to $T^{2}$. Moreover, it is proved that for each odd $q$, there is such a $T$ commuting with a transformation of order $q$. For any $n$, we show the existence of a weakly mixing $T$ conjugate to $T^{2}$ and whose rank is finite and greater than $n$.
\end{abstract}

0. Introduction. Recently there has been progress in studying ergodic transformations isomorphic to their composition squares [Ag2], [Go2], [Go3] (see also [Go1]). In [Ag2], Ageev answered a well known question: he proved the existence of a weakly mixing rank-one transformation $T$ conjugate to $T^{2}$. However, his proof given in the Baire category framework is not constructive. Thus, no concrete example of $T$ is known so far. The main purpose of the present paper is to construct such a $T$ via the cutting-and-stacking algorithm with explicitly described spacers. For this, we apply a group action approach suggested first by A. del Junco in [dJ3] to produce a counterexample in the theory of simple actions. The idea is to select an auxiliary countable group $H$ and an element $h \in H$ and to construct via $(C, F)$-techniques a special funny rank-one action $V$ of $H$ in such a way that the transformation $V_{h}$ has required dynamical properties. In our case, $H$ is the group of 2-adic rationals and $h=1$. The action $V$ is constructed in $\S 2$. For other - sometimes unexpected - applications of the group action approach we refer to [Ma], [Ag1], [Da5], [Da6], [DdJ]. For the basics of the cutting-and-stacking $(C, F)$ techniques we refer to $\S 2$ below (see also [Da3], [Da4], [DaS] and a survey [Da7]).

A new short category proof of the existence theorem from [Ag2] is given below, in Section 1 (Theorem 1.3). Section 2 contains the main result of the present paper (Theorem 2.2). In Section 3 we discuss "elements" of the general theory of ergodic transformations $T$ conjugate to $T^{2}$ : generic aspects of

2000 Mathematics Subject Classification: Primary 37A40; Secondary 37A15, 37A20, $37 \mathrm{~A} 30$.

Key words and phrases: $(C, F)$-construction, rank-one transformation. 
the inclusion of $T$ into actions of some related "larger" groups, application of the co-inducing procedure from $[\mathrm{D}-\mathrm{S}]$ and some spectral properties of $T$. In Section 4, given $T$ conjugate to $T^{2}$, we investigate which ergodic compact extensions of $T$ are conjugate to their square and what is the structure of the conjugating maps. As an application, we show that for any $n$, there exists a weakly mixing transformation $T$ conjugate to $T^{2}$ and such that the rank of $T$ is finite and greater than $n$ (Theorem 4.10). In [Go2] Goodson isolated the class of those $T$ (conjugate to $T^{2}$ ) that have King's weak closure property [Ki1]. Such $T$ do not commute with periodic transformations of even order. He raised the question whether they can commute with periodic transformations of odd order. We answer this affirmatively using Baire category arguments: for any odd $q>0$, there exists a weakly mixing rank-one transformation $T$ conjugate to $T^{2}$ and whose centralizer contains an element of order $q$ (Theorem 4.9). Concrete examples can also be constructed via the $(C, F)$-techniques as in Theorem 2.2. We also note that some results from [Go2] and [Go3] are refined and generalized in Sections 3 and 4. In the final Section 5 we state several open problems.

1. Notation and short proof of Ageev's theorem. Let $(X, \mathfrak{B}, \mu)$ be a standard non-atomic probability space. Denote by $\operatorname{Aut}_{0}(X, \mu)$ the group of $\mu$-preserving transformations of $X$. It is well known that $\operatorname{Aut}_{0}(X, \mu)$ endowed with the weak (operator) topology is a Polish group [Ha].

We recall that a transformation $T \in \operatorname{Aut}_{0}(X, \mu)$ has rank $n$ (we will denote that by $\operatorname{rk}(T)=n$ ) if $n$ is the smallest $r \in \mathbb{N}$ such that there exist measurable subsets $B_{j}^{(m)}$ and positive integers $h_{j}^{(m)}$ such that the subsets $T^{i} B_{j}^{(m)}, j=1, \ldots, r, i=1, \ldots, h_{j}^{(m)}$, are pairwise disjoint and approximate the entire $\sigma$-algebra $\mathfrak{B}$ as $m \rightarrow \infty$. The latter means that given $B \in \mathfrak{B}$, there are subsets $A^{(m)}$ such that $\nu\left(B \triangle A^{(m)}\right) \rightarrow 0$ and every $A^{(m)}$ is the union of several subsets $T^{i} B_{j}^{(m)}$ with $0 \leq j \leq r$ and $1 \leq i \leq h_{j}^{(m)}$.

It follows from the classical results of Katok and Stepin $[\mathrm{KaS}]$ that the subset $\mathcal{R}^{1}$ of rank-one transformations is residual in $\operatorname{Aut}_{0}(X, \mu)$. We need the following a bit subtler fact.

Proposition 1.1. $\mathcal{R}^{1}$ is a $G_{\delta}$ in $\operatorname{Aut}_{0}(X, \mu)$.

Proof. Take a countable subfamily $\mathfrak{B}_{0} \subset \mathfrak{B}$ which is dense in $(\mathfrak{B}, \mu)$. Let $B_{1}, B_{2}, \ldots$ be a sequence of measurable subsets in $X$ such that each element of $\mathfrak{B}_{0}$ occurs infinitely often in this sequence. Now given $A \in \mathfrak{B}, n, m \in \mathbb{N}$ and $\varepsilon, \delta>0$, we let

$$
\begin{aligned}
\mathcal{O}_{A, n, \varepsilon} & :=\left\{T \in \operatorname{Aut}_{0}(X, \mu) \mid \mu\left(T^{j} A \cap T^{k} A\right)<\varepsilon \mu(A) / n \text { for all } 0 \leq j, k<n\right\}, \\
\mathcal{O}_{A, n, \varepsilon}^{m, \delta} & :=\left\{T \in \mathcal{O}_{A, n, \varepsilon} \mid \max _{0 \leq i<m} \min _{J \subset\{0, \ldots, n\}} \mu\left(B_{i} \triangle \bigcup_{j \in J} T^{j} A\right)<\delta\right\} .
\end{aligned}
$$


Clearly, the two subsets are open in $\operatorname{Aut}_{0}(X, \mu)$. It remains to notice that

$$
\mathcal{R}^{1}=\bigcap_{k=1}^{\infty} \bigcup_{A \in \mathfrak{B}_{0}} \bigcup_{n=1}^{\infty} \mathcal{O}_{A, n, 1 / k}^{k, 1 / k} .
$$

From now on let $H$ stand for the group of 2-adic rationals. We denote by $G$ the semidirect product $H \rtimes \mathbb{Z}$ with the multiplication

$$
(h, n)\left(h^{\prime}, n^{\prime}\right):=\left(h+2^{n} h^{\prime}, n+n^{\prime}\right), \quad h, h^{\prime} \in H, n, n^{\prime} \in \mathbb{Z} .
$$

Let $\mathcal{A}_{G}$ stand for the set of $\mu$-preserving $G$-actions $V=\left(V_{g}\right)_{g \in G}$ on $X$. Of course, there is a one-to-one correspondence between $\mathcal{A}_{G}$ and the pairs of transformations $T, S \in \operatorname{Aut}_{0}(X, \mu)$ such that $S T S^{-1}=T^{2}$. The correspondence is given by the formulae $V_{(1,0)}:=T$ and $V_{(0,1)}:=S$.

LEMMA 1.2. If $T$ is ergodic then $V$ is free.

Proof. Suppose first that there is a subset $A \subset X$ with $\mu(A)>0$ such that $V_{h} x=x$ for all $x \in X$ and some $h \in H, h \neq 0$. Since $V_{h}$ and $T$ commute and $T$ is ergodic, $V_{h}=\mathrm{Id}$. This implies in turn that a power of $T$ is the identity, a contradiction. Thus the $H$-subaction $\left(V_{h}\right)_{h \in H}$ is free. Suppose now that there are $h \in H$ and $n \in \mathbb{N} \backslash\{0\}$ with $V_{h} x=S^{n} x$ on a set of positive measure. Since the transformation $S^{n}$ normalizes the ergodic transformation group $\left\{V_{h} \mid h \in H\right\}$, there exists a measurable map $m: X \rightarrow H$ such that $S^{n} x=V_{m(x)} x$ for a.a. $x \in X$. Hence the relation $S^{n} T=T^{2^{n}} S^{n}$ plus the freeness of $\left(V_{h}\right)_{h \in H}$ yield now $m(T x)+1=m(x)+2^{n}$ for a.a. $x \in X$. Then for each $\lambda \in \mathbb{R}$, the function $X \ni x \mapsto \exp (2 \pi i \lambda m(x)) \in \mathbb{T}$ is an eigenfunction of $T$ corresponding to the eigenvalue $\exp \left(2 \pi i \lambda\left(2^{n}-1\right)\right)$. Since the discrete spectrum of $T$ is at most countable, we obtain a contradiction.

Now we are ready to give a short proof of the main result from [Ag2].

THEOREM 1.3. There exists a weakly mixing rank-one transformation conjugate to its square.

Proof. Note that $\mathcal{A}_{G}$ is a closed subset of the infinite product space $\operatorname{Aut}_{0}(X, \mu)^{G}$ furnished with the product topology. Moreover, $\operatorname{Aut}_{0}(X, \mu)$ acts continuously on $\mathcal{A}_{G}$ by conjugation as follows: $(T \cdot V)_{g}:=T V_{g} T^{-1}$, $g \in G$. We let

$$
\begin{aligned}
& \mathcal{W}_{G}:=\left\{V \in \mathcal{A}_{G} \mid V_{(1,0)} \text { is weakly mixing }\right\}, \\
& \mathcal{R}_{G}^{1}:=\left\{V \in \mathcal{A}_{G} \mid V_{(1,0)} \in \mathcal{R}^{1}\right\} .
\end{aligned}
$$

Since the set of weakly mixing transformations and the set of rank-one transformations are both $G_{\delta}$ in $\operatorname{Aut}_{0}(X, \mu)$ (see [Gl] and Proposition 1.1) and the map $\mathcal{A}_{G} \ni V \mapsto V_{(1,0)} \in \operatorname{Aut}_{0}(X, \mu)$ is continuous, it follows that $\mathcal{W}_{G}$ and $\mathcal{R}_{G}^{1}$ are both $G_{\delta}$ in $\mathcal{A}_{G}$. We notice that $\mathcal{W}_{G}$ contains any Bernoullian shiftwise $G$-action. Any such action is free. Let us show that $\mathcal{R}_{G}^{1}$ also 
contains a free $G$-action. Since $H$ is the inductive limit of the sequence $\mathbb{Z} \stackrel{\times 2}{\longrightarrow} \mathbb{Z} \stackrel{\times 2}{\longrightarrow} \cdots$, we can represent the compact dual group $\widehat{H}$ as the inverse limit of the sequence $\mathbb{T} \leftarrow \mathbb{T} \leftarrow \cdots$, where all the arrows denote the power two homomorphism. Take any $z_{0} \in \mathbb{T}$ of infinite order and consider the infinite sequence $k^{\prime}:=\left(z_{0}, z_{1}, \ldots\right) \in \widehat{H}$ with $z_{n}=z_{n+1}^{2} \in \mathbb{T}$ for all $n$. Now we define two measure preserving transformations of $\left(\widehat{H}, \lambda_{\widehat{H}}\right)$ by setting $T k:=k k^{\prime}$ and $S k=k^{2}$. Then $S T S^{-1}=T^{2}$. Moreover, $T$ is ergodic by the standard criterion of ergodicity for rotations on compact groups [CFS]. By Lemma 1.2, the action $V$ of $G$ corresponding to $(T, S)$ is free. Since $T$ has pure point spectrum, $T \in \mathcal{R}^{1}$ by [dJ1], i.e. $V \in \mathcal{R}_{G}^{1}$.

By [FoW], the $\operatorname{Aut}_{0}(X, \mu)$-orbit of any free $G$-action is dense in $\mathcal{A}_{G}$. Since $\mathcal{W}_{G}$ and $\mathcal{R}_{G}^{1}$ are $\operatorname{Aut}_{0}(X, \mu)$-invariant, it follows that they are both residual in $\mathcal{A}_{G}$. Hence their intersection is non-empty.

Notice that the above example of an affine $G$-action $V \in \mathcal{R}_{G}^{1}$ appeared first (in a slightly different form) in [Go2]. It was reproduced later in [Ag2].

2. Explicit examples. We start this section by recalling the $(C, F)$ construction (see also [dJ3], [Da3]-[Da7], [DaS], [DdJ]). Let $\left(C_{m}\right)_{m=1}^{\infty}$ and $\left(F_{m}\right)_{m=0}^{\infty}$ be two sequences of finite subsets in $H$ such that for each $m \geq 0$ the following properties are satisfied:

$$
F_{m}+C_{m+1} \subset F_{m+1}, \quad \# C_{m+1}>1,
$$
the sets $F_{m}+c, c \in C_{m+1}$, are pairwise disjoint.

We put $X_{m}:=F_{m} \times C_{m+1} \times C_{m+2} \times \cdots$, endow $X_{m}$ with the (compact) product topology and define a continuous embedding $X_{m} \rightarrow X_{m+1}$ by setting

$$
\left(f_{m}, c_{m+1}, c_{m+2}, \ldots\right) \mapsto\left(f_{m}+c_{m+1}, c_{m+2}, \ldots\right) .
$$

Then we have $X_{1} \subset X_{2} \subset \cdots$. Let $X:=\bigcup_{m} X_{m}$ stand for the topological inductive limit of the sequence $X_{m}$. Clearly, $X$ is a locally compact totally disconnected metrizable space without isolated points and $X_{m}$ is clopen in $X$. Hence the corresponding Borel $\sigma$-algebra $\mathfrak{B}$ is standard. Assume in addition that

$$
\prod_{m=1}^{\infty} \frac{\# F_{m+1}}{\# F_{m} \# C_{m+1}}<\infty
$$

Then it is easy to see that there exists a unique probability measure $\mu$ on $(X, \mathfrak{B})$ such that the restriction of $\mu$ to each $X_{m}$ is the infinite product measure

$$
\tau_{m} \times \lambda_{m+1} \times \lambda_{m+2} \times \cdots,
$$

where $\lambda_{j}$ is the equidistribution on $C_{j}$ and $\tau_{m}$ is a finite measure on $F_{m}$ with $\tau_{m}(f)=\tau_{m}\left(f^{\prime}\right)$ for all $f, f^{\prime} \in F_{m}$. Thus $(X, \mathfrak{B}, \mu)$ is a standard probability 
space. Given $h \in H$ and $m>0$, we set

$$
D_{h}^{(m)}:=\left(F_{m} \cap F_{m}-h\right) \times C_{m+1} \times C_{m+2} \times \cdots \quad \text { and } \quad R_{h}^{(m)}:=D_{-h}^{(m)} .
$$

Clearly, $D_{h}^{(m)}$ and $R_{h}^{(m)}$ are clopen subsets of $X_{n}$. Moreover, $D_{h}^{(m)} \subset D_{h}^{(m+1)}$ and $R_{h}^{(m)} \subset R_{h}^{(m+1)}$. Define a map $T_{h}^{(m)}: D_{h}^{(m)} \rightarrow R_{h}^{(m)}$ by setting

$$
T_{h}^{(m)}\left(f_{m}, c_{m+1}, \ldots\right):=\left(h+f_{m}, c_{m+1}, \ldots\right) .
$$

Clearly, it is a homeomorphism. Put

$$
D_{h}:=\bigcup_{m=1}^{\infty} D_{h}^{(m)} \quad \text { and } \quad R_{h}:=\bigcup_{m=1}^{\infty} R_{h}^{(m)}=D_{h^{-1}} .
$$

Then $D_{h}$ and $R_{h}$ are open subsets of $X$. Moreover, a homeomorphism $T_{h}: D_{h} \rightarrow R_{h}$ is well defined by $T_{h}\left\lceil D_{h}^{(m)}=T_{h}^{(m)}\right.$ for all $m$. Suppose now that

$$
\left(F_{m}\right)_{m \geq 0} \text { is a Følner sequence in } G \text {. }
$$

This implies $\mu\left(D_{h}^{(m)}\right) \rightarrow 1$ as $m \rightarrow \infty$. Hence $\mu\left(D_{h}\right)=\mu\left(R_{h}\right)=1$. Since $\mu(O)>0$ for each open subset $O \subset X$, it follows that the subset $D:=$ $\bigcap_{h \in H} D_{h}=\bigcap_{h \in H} R_{h}$ is a dense $G_{\delta}$ of full $\mu$-measure. It is easy to see that $T_{h_{2} h_{1}}=T_{h_{2}} T_{h_{1}}$ on $D$ for all $h_{1}, h_{2} \in H$. Thus $T:=\left(T_{h}\right)_{h \in H}$ is a continuous $H$-action on the Polish (in the induced topology) space $D$. This action is minimal. To see this, just notice that for any $m$,

(i) the $T$-orbit equivalence relation restricted to $X_{m} \cap D$ coincides with the restriction of the tail equivalence relation $\mathcal{T}_{m}$ on the infinite product space $X_{m}$ to $D$,

(ii) $D \cap X_{m}$ is invariant under $\mathcal{T}_{m}$.

Moreover, $T$ preserves $\mu$ and $T$ is free and ergodic.

Definition 2.1. We call $T$ the $(C, F)$-action of $H$ associated to $\left(C_{m+1}, F_{m}\right)_{m=0}^{\infty}$.

In the following we will not distinguish between sets, maps, and transformations which agree a.e. For each subset $A \subset F_{m}$, we let

$$
[A]_{m}:=\left\{x=\left(f_{m}, c_{m+1}, \ldots\right) \in X_{m} \mid f_{m} \in A\right\}
$$

and call it an $m$-cylinder. Then

$$
\begin{aligned}
& {[A \cap B]_{m}=[A]_{m} \cap[B]_{m} \quad \text { and } \quad[A \cup B]_{m}=[A]_{m} \cup[B]_{m},} \\
& {[A]_{m}=\left[A+C_{m+1}\right]_{m+1}=\bigsqcup_{c \in C_{m+1}}[A+c]_{m+1},} \\
& T_{h}[A]_{m}=[h+A]_{m} \quad \text { if } h+A \subset F_{m}, \\
& \mu\left([A]_{m}\right)=\# C_{m+1} \cdot \mu\left([A+c]_{m+1}\right) \quad \text { for every } c \in C_{m+1}, \\
& \mu\left([A]_{m}\right)=\mu\left(X_{m}\right) \frac{\# A}{\# F_{m}},
\end{aligned}
$$


where $\sqcup$ denotes the union of mutually disjoint sets. Moreover, given $Y \in \mathfrak{B}$,

$$
\min _{A \subset F_{m}} \mu\left(Y \triangle[A]_{m}\right) \rightarrow 0 \quad \text { as } m \rightarrow \infty .
$$

This means that $T$ has funny rank one [Da3].

To construct a special $(C, F)$-action of $H$ we need several definitions. Let $F=\left\{2^{-k}, 2 \cdot 2^{-k}, \ldots, L-2^{-k}\right\} \subset H$ for some $k, L \in \mathbb{N}$. Then $F$ is an arithmetic progression of length $2^{k} L-1$. It is easy to see that for each $0 \leq i<\# F$, there is a unique element $f_{i} \in F$ such that

$$
\frac{1}{2^{k}}+i=f_{i}+m_{i}\left(L-\frac{1}{2^{k}}\right)
$$

where $m_{i}$ is a non-negative integer with $m_{i} \leq 2^{k}$. Moreover, the map $i \mapsto f_{i}$ is one-to-one and hence $F=\left\{f_{i} \mid i=0, \ldots, \# F-1\right\}$. Now for a given $p>0$, we define the $p$-good tiling set $C$ for $F$ by setting

$$
C:=\left\{0,-2^{-k-1}\right\}+\left\{0, L-2^{-k}, \ldots,\left(2^{k} p-1\right)\left(L-2^{-k}\right)\right\}
$$

Then $(F+c) \cap\left(F+c^{\prime}\right)=\emptyset$ whenever $c \neq c^{\prime} \in C$. The sum $F+C$ equals the arithmetic progression $\left\{2^{-k-1}, 2 \cdot 2^{-k-1}, \ldots, 2^{k} p\left(L-2^{-k}\right)\right\}$. We now set

$$
C^{\prime}:=\left\{0,-2^{-k-1}\right\}+\left\{0, L-2^{-k}, \ldots,\left(2^{k}(p-1)-1\right)\left(L-2^{-k}\right)\right\}
$$

and call it the reduced p-good tiling set for $F$. It follows from (2-5) and the inequality $m_{i} \leq 2^{k}$ that

$$
\frac{\# C^{\prime}}{\# C}=1-\frac{1}{p} \quad \text { and } \quad i+2^{-k}+C^{\prime} \subset f_{i}+C
$$

for all $0 \leq i<\# F$.

Consider now another case. Suppose that $F=\left\{2^{-k}, 2 \cdot 2^{-k}, \ldots, L\right\}$ for some integers $k, L>0$. Then we define the Chacon tiling set $C$ for $F$ by setting

$$
C:=\{0, L, 2 L+1\} .
$$

Of course, $(F+c) \cap\left(F+c^{\prime}\right)=\emptyset$ whenever $c \neq c^{\prime} \in C$.

Now we are going to construct a special $(C, F)$-action $T=\left(T_{h}\right)_{h \in H}$ of $H$. Fix a sequence of positive integers $p_{n}$ such that $\sum_{n=1}^{\infty} p_{n}^{-1}<\infty$. Then choose $\left(C_{n}\right)_{n=1}^{\infty}$ and $\left(F_{n}\right)_{n=0}^{\infty}$ recurrently as follows:

- $F_{0}=\{0.5\}$,

- $C_{2 n+1}$ is a $p_{n}$-good tiling for $F_{2 n}$,

- $F_{2 n+1}$ is the shortest arithmetic progression which contains the arithmetic progression $F_{2 n}+C_{2 n+1}$ and satisfies the following two conditions: the largest element of $F_{2 n+1}$ is an integer and the cardinality of the subset $C_{2 n+1}^{+}:=\left\{c \in C_{2 n+1} \mid 2 \cdot F_{2 n}+2 c \subset F_{2 n+1}\right\}$ is $0.5 \# C_{2 n+1}$,

- $C_{2 n+2}$ is the Chacon tiling set for $F_{2 n+1}$, 
- $F_{2 n+2}$ is the shortest arithmetic progression of positive 2-adic rationals such that $F_{2 n+1}+C_{2 n+2} \subset F_{2 n+2}$ and the sum max $F_{2 n+2}+\min F_{2 n+2}$ is an integer.

Now it is easy to see that the conditions $(2-1)-(2-4)$ are all satisfied. Hence the associated rank-one $(C, F)$-action $T=\left(T_{h}\right)_{h \in H}$ is well defined.

THEOREM 2.2. The transformation $T_{1}$ is weakly mixing and of rank one. Moreover, $T_{1}$ is conjugate to $T_{2}$.

Proof. We first prove that $T_{1}$ is weakly mixing. Let $e: X \rightarrow \mathbb{T}$ be an eigenfunction of $T_{1}$ corresponding to a non-trivial eigenvalue $\lambda \in \mathbb{T}$, i.e. $e \circ T_{1}=\lambda e,|e| \equiv 1$ and $\lambda \neq 1$. Given $\varepsilon>0$, there is a subset $A \subset X$ of positive measure such that

$$
|e(x)-e(y)|<\varepsilon \quad \text { for all } x, y \in A .
$$

Now we can find $n>0$ and a cylinder $[f]_{2 n+1}$ such that

$$
\mu\left(A \cap[f]_{2 n+1}\right)>0.99 \mu\left([f]_{2 n+1}\right) .
$$

We recall that $F_{2 n+1}=\left\{2^{-k_{n}}, 2 \cdot 2^{-k_{n}}, \ldots, L_{n}\right\}$ and $C_{2 n+2}=\left\{0, L_{n}, 2 L_{n}+1\right\}$ for some positive integers $k_{n}$ and $L_{n}$. Since $[f]_{2 n+1}$ is the union of the three disjoint subsets $[f]_{2 n+2},\left[f+L_{n}\right]_{2 n+2}$ and $\left[f+2 L_{n}+1\right]_{2 n+2}$ of equal measure, and $\left[f+L_{n}\right]_{2 n+2}=T_{L_{n}}[f]_{2 n+2}$ and $\left[f+2 L_{n}+1\right]_{2 n+2}=T_{2 L_{n}+1}[f]_{2 n+2}$, we deduce from (2-8) that there exists a subset $B \subset[f]_{2 n+2}$ of positive measure such that $B \sqcup T_{L_{n}} B \sqcup T_{2 L_{n}+1} B \subset A$. Hence by $(2-7)$,

$\left|1-\lambda^{L_{n}}\right|=\left|e(b)-e\left(T_{L_{n}} b\right)\right|<\varepsilon \quad$ and $\quad\left|1-\lambda^{2 L_{n}+1}\right|=\left|e(b)-e\left(T_{2 L_{n}+1} b\right)\right|<\varepsilon$ for all $b \in B$. This yields $|\lambda-1|<3 \varepsilon$. Since $\varepsilon$ is arbitrary, we deduce that $\lambda=1$, a contradiction.

Now we will show that $T_{1}$ has rank one. Recall that for each $n>0$, there exist positive integers $d_{n}$ and $R_{n}$ such that $F_{2 n}=\left\{2^{-d_{n}}, 2 \cdot 2^{-d_{n}}, \ldots\right.$ $\left.\ldots, R_{n}-2^{-d_{n}}\right\}$. We let $D_{n}:=\left[2^{-d_{n}}+C_{2 n+1}^{\prime}\right]_{2 n+1}$, where $C_{2 n+1}^{\prime} \subset C_{2 n+1}$ is the reduced $p_{n}$-good tiling set for $F_{2 n}$. It follows from (2-6) that $T_{i} D_{n} \subset$ $\left[f_{i}\right]_{2 n}$ for all $i=0, \ldots, \# F_{2 n}-1$, where $\left\{f_{i}\right\}_{i=0}^{\# F_{2 n}-1}$ is an enumeration of the elements of $F_{2 n}$. Moreover, (2-6) yields

$$
\sum_{i=0}^{\# F_{2 n}-1} \mu\left(\left[f_{i}\right]_{2 n} \backslash T_{i} B_{n}\right)=\sum_{i=0}^{\# F_{2 n}-1} \mu\left(\left[C_{2 n+1} \backslash C_{2 n+1}^{\prime}\right]_{2 n+1}\right)<p_{n}^{-1} .
$$

Since the sequence of towers $[f]_{2 n}, f \in F_{2 n}$, approximates the entire $\sigma$ algebra $\mathfrak{B}$ by (2-4), it now follows that the sequence of towers $T_{i} B_{n}, i=$ $0, \ldots, \# F_{2 n}-1$, also approximates $\mathfrak{B}$ as $n \rightarrow \infty$. Hence $T_{1}$ is of rank one.

It remains to establish that $T_{1}$ is conjugate to its square. To define a transformation $S$ which conjugates $T_{1}$ and $T_{2}$ it suffices to show how it acts on the cylinders. For each $n>0$, we put $C_{2 n+1}^{-}:=C_{2 n+1} \backslash C_{2 n+1}^{+}$. Now 
given $f \in F_{2 n}$, we partition the $2 n$-cylinder $[f]_{2 n}$ into two $(2 n+1)$-cylinders $\left[f+C_{2 n+1}^{+}\right]_{2 n+1}$ and $\left[f+C_{2 n+1}^{-}\right]_{2 n+1}$ of equal measure and define the image of the first one under $S$ by setting

$$
S\left[f+C_{2 n+1}^{+}\right]_{2 n+1}:=\left[2 f+2 \cdot C_{2 n+1}^{+}\right]_{2 n+1} .
$$

It is well defined due to our choice of $F_{2 n+1}$. Next, we partition the second $(2 n+1)$-cylinder into two $(2 n+3)$-cylinders

$$
\left[f+C_{2 n+1}^{-}+C_{2 n+2}+C_{2 n+3}^{+}\right]_{2 n+3} \text { and }\left[f+C_{2 n+1}^{-}+C_{2 n+2}+C_{2 n+3}^{-}\right]_{2 n+3}
$$

of equal measure and define the image of the first one under $S$ by setting $S\left[f+C_{2 n+1}^{-}+C_{2 n+2}+C_{2 n+3}^{+}\right]_{2 n+3}:=\left[2 f+2 \cdot C_{2 n+1}^{-}+2 \cdot C_{2 n+2}+2 \cdot C_{2 n+3}^{+}\right]_{2 n+3}$. Continuing this procedure infinitely many times, we define the $S$-image of the entire $[f]_{2 n}$, since

$$
[f]_{2 n}=\bigsqcup_{n \leq q<\infty}\left[f+\sum_{j=n}^{q-1}\left(C_{2 j+1}^{-}+C_{2 j+2}\right)+C_{2 q+1}^{+}\right]_{2 q+1} .
$$

It is straightforward that $\mu\left(S[f]_{2 n}\right)=\mu\left([f]_{2 n}\right)$ and $S[f]_{2 n} \cap S\left[f^{\prime}\right]_{2 n}=\emptyset$ whenever $f \neq f^{\prime} \in F_{2 n}$. Hence $S \in \operatorname{Aut}_{0}(X, \mu)$. Moreover, it is easy to see that $S T_{1}=T_{2} S$, as desired.

REMARK 2.3. It is easy to deduce from the proof of Theorem 2.1 that $T_{\# F_{2 n}} D_{n} \subset\left[f_{0}\right]_{2 n}$. This implies $T_{1}^{\# F_{2 n}} \rightarrow \operatorname{Id}$ as $n \rightarrow \infty$. Hence $T_{1}$ is rigid.

REMARK 2.4. Slightly modifying the construction of $T$ one can produce explicitly a rigid weakly mixing rank-one transformation $R$ conjugate to $R^{j}$ for any $j>1$. Moreover, the corresponding conjugating maps will pairwise commute. The existence of such a transformation can also be proved via Baire category arguments as in Theorem 1.3. The only difference in the proof is to consider the group $\mathbb{Q} \rtimes_{\alpha} \bigoplus_{j=1}^{\infty} \mathbb{Z}$ instead of $G$, where $\alpha$ denotes the following action of $\bigoplus_{j=1}^{\infty} \mathbb{Z}$ on $\mathbb{Q}$ :

$$
\alpha\left(n_{1}, \ldots, n_{k}, 0,0, \ldots\right) q:=2^{n_{1}} \cdot 3^{n_{2}} \cdots(k+1)^{n_{k}} \cdot q .
$$

3. Extending transformations to $G$-actions. Let $T$ be an ergodic transformation of $(X, \mathfrak{B}, \mu)$. We consider the problem of whether $T$ is conjugate to its square, i.e. $S T S^{-1}=T^{2}$. This can be "split" into two separate subproblems:

(o) Does $T$ embed into a $\mu$-preserving action $H$-action $V=\left(V_{h}\right)_{h \in H}$ on the same measure space (we mean that $V_{1}=T$ )?

( $\widehat{o})$ Given an $H$-action $V$, does it extend to a $\mu$-preserving $G$-action on the same measure space? 
Notice that the affirmative answer to $(0)$ is stronger than the fact that $T$ has roots of order $2^{n}$ for each $n>0$. The corresponding counterexample was produced via the $(C, F)$-techniques in [Ma].

Some necessary and sufficient conditions for extending an action of a subgroup to an action of a "larger" group are given in [Da2, Proposition 2.5]. However, being of rather abstract orbital nature they are of little practical importance.

We now answer (o) and ( $\widehat{o})$ generically, i.e. up to a meager subset in the Baire category sense. Let $\mathcal{A}_{G}$ and $\mathcal{A}_{H}$ denote the Polish spaces of $\mu$-preserving $G$-actions and $H$-actions respectively. They are $\operatorname{Polish}_{\operatorname{Aut}_{0}}(X, \mu)$ spaces, and the natural projections

$$
\pi_{G}: \mathcal{A}_{G} \ni W \mapsto\left(W_{h}\right)_{h \in H} \in \mathcal{A}_{H}, \quad \pi_{H}: \mathcal{A}_{H} \ni V \mapsto V_{1} \in \operatorname{Aut}_{0}(X, \mu)
$$

are both continuous and $\operatorname{Aut}_{0}(X, \mu)$-equivariant (see $\S 1$ ). It follows from [dRdS] that

$(\bullet)$ a generic transformation from $\operatorname{Aut}_{0}(X, \mu)$ embeds into an $H$-action.

On the other hand,

$(\widehat{\bullet})$ the subset $\pi_{G}\left(\mathcal{A}_{G}\right)$ of $H$-actions extending to $G$-actions is meager in $\mathcal{A}_{H}$.

Indeed, if $(\widehat{\bullet})$ does not hold then $\pi_{G}\left(\mathcal{A}_{G}\right)$ is residual in $\mathcal{A}_{H}$ by the $0-1$ law from $[\mathrm{GlK}]$ (see also $[\mathrm{FoW}]$ ). It follows from the argument presented in [dRdS, §3.2] that the locally dense points for $\pi_{H}$ are dense in $\mathcal{A}_{H}$. Hence the set $\pi_{H}\left(\pi_{G}\left(\mathcal{A}_{G}\right)\right)$ is residual in $\operatorname{Aut}_{0}(X, \mu)$ [Ki2]. However, this contradicts [dJ2], where it was shown that a generic transformation is disjoint from (hence, not conjugate to) its square.

We think that it is relevant to mention here that an important class of transformations conjugate to their squares - considered in [Go2] and [Ag2] can be described in a canonical way via co-inducing. We recall the definition following $[\mathrm{D}-\mathrm{S}]$.

Definition 3.1. Let $\Gamma$ be a countable group and $\Lambda$ a subgroup of $\Gamma$. Let $\widehat{T}=\left(\widehat{T}_{h}\right)_{h \in \Lambda}$ be a measure preserving action of $\Lambda$ on a standard probability space $(Y, \mathfrak{C}, \nu)$. Select a cross-section $\sigma: \Lambda \backslash \Gamma \rightarrow \Gamma$ of the quotient map $\Gamma \rightarrow \Lambda \backslash \Gamma$ with $\sigma(\Lambda)=1_{\Gamma}$. Define an action $T=\left(T_{g}\right)_{g \in \Gamma}$ of $\Gamma$ on the product space $(X, \mathfrak{B}, \mu):=(Y, \mathfrak{C}, \nu)^{\Lambda \backslash \Gamma}$ by setting

$$
\left(T_{g} x\right)\left(\Lambda g^{\prime}\right):=\widehat{T}_{\sigma\left(\Lambda g^{\prime}\right) g \sigma\left(\Lambda g^{\prime} g\right)^{-1}} x\left(\Lambda g^{\prime} g\right)
$$

for all maps $x: \Lambda \backslash \Gamma \rightarrow Y$ and $g \in \Gamma$. Then $T$ is said to be co-induced from $\widehat{T}$.

It is easy to see that $T$ does not depend (up to conjugacy) on the choice of $\sigma$. Moreover, if $\widehat{T}$ is free or ergodic then so is $T$. Notice that $T$ is defined on a "larger" space than $Y$ : the original action $\widehat{T}$ is only a factor of $\left(T_{h}\right)_{h \in \Lambda}$. 
Suppose now that we are given an action $\widehat{T}$ of $H$ on $(Y, \mathfrak{C}, \nu)$. Then the co-induced action $T$ of $G$ is defined on $(Y, \nu)^{\mathbb{Z}}$. The generators $(1,0)$ and $(0,1)$ of $G$ act as follows:

$$
\left(T_{(1,0)} y\right)_{n}=\widehat{T}_{2^{n}} y_{n} \quad \text { and } \quad\left(T_{(0,1)} y\right)_{n}=y_{n+1}, \quad n \in \mathbb{Z} .
$$

Thus we obtain the well-known examples from [Go2] and [Ag2].

It is interesting to note that $(\bullet)$ combined with [Ag1] implies that a generic transformation $T \in \operatorname{Aut}_{0}(X, \mu)$ embeds into an $H$-action $\widehat{V}$ such that the $(1,0)$-transformation $V_{(1,0)}$ of the corresponding $G$-action $V$ coinduced from $\widehat{H}$ is weakly mixing and has a simple spectrum. However-as noticed by V. Ryzhikov [Ry] - it is never of rank one (cf. the example in Theorem 2.2). Indeed, if $V_{(1,0)}$ were of rank one then its factor $T \times T^{2}$ would also be of rank one. Hence by King's weak closure theorem [Ki1] the transformation Id $\times T$ commuting with $T \times T^{2}$ is the weak limit of a sequence of powers of $T \times T^{2}$. Thus Id $=\lim _{i \rightarrow \infty} T^{n_{i}}$ and $T=\lim _{i \rightarrow \infty} T^{2 n_{i}}$, a contradiction.

Now we are going to discuss some aspects of spectral theory for the transformations embedding into $G$-actions (or, equivalently, transformations conjugate to their squares). Let $S T S^{-1}=T^{2}$ and $V=\left(V_{g}\right)_{g \in G}$ stand for the corresponding $G$-action. Denote by $U: G \ni g \mapsto U_{g} \in \mathcal{U}\left(L^{2}(X, \mu)\right)$ the Koopman unitary representation of $G$ associated to $G$. Then by the spectral theorem for $U\lceil H$, there exist a probability measure $\sigma$ on $\widehat{H}$ and a Borel map $m: \widehat{H} \ni w \mapsto m(w) \in \mathbb{N} \cup\{\infty\}$ such that the following decomposition holds (up to unitary equivalence):

$$
L^{2}(X, \mu)=\int_{\widehat{H}}^{\oplus} \mathcal{H}_{w} d \sigma(w) \quad \text { and } \quad U_{h}=\int_{\widehat{H}} w(h) I_{w} d \sigma(w),
$$

for each $h \in H$, where $w \mapsto \mathcal{H}_{w}$ is a Borel field of Hilbert spaces, $\operatorname{dim} \mathcal{H}_{w}=$ $m(w)$ and $I_{w}$ is the identity operator on $\mathcal{H}_{w}$. Let $Q$ denote the power two automorphism $w \mapsto w^{2}$ of $\widehat{H}$. Since the unitary representation $H \ni h \mapsto$ $U_{2 h} \in \mathcal{U}\left(L^{2}(X, \mu)\right)$ of $H$ is unitarily equivalent to $U\left\lceil H\right.$ via $U_{(0,1)}$, it follows that $\sigma$ is quasi-invariant under $Q$ and $m \circ Q=m$.

The canonical embedding $\mathbb{Z} \subset H$ induces a projection $\pi: \widehat{H} \rightarrow \mathbb{T}$. Let $\sigma=\int_{\mathbb{T}} \sigma_{z} d \widehat{\sigma}(z)$ denote the desintegration of $\sigma$ relative to this projection. Then we derive from (3-1) the spectral decomposition for the operator $U_{(1,0)}$ :

$$
L^{2}(X, \mu)=\int_{\mathbb{T}}^{\oplus} \mathcal{H}_{z}^{\prime} d \widehat{\sigma}(z) \quad \text { and } \quad U_{(1,0)}=\int_{\mathbb{T}} z I_{z} d \widehat{\sigma}(z)
$$


where $\mathcal{H}_{z}^{\prime}:=\int_{\widehat{H}}^{\oplus} \mathcal{H}_{w} d \sigma_{z}(w)$. Let $l(z):=\operatorname{dim} \mathcal{H}_{z}^{\prime}, z \in \mathbb{T}$. Then

$$
l(z)= \begin{cases}\infty & \text { if } \sigma_{z} \text { is not purely atomic, } \\ \sum_{\sigma_{z}(w)>0} m(w) & \text { otherwise. }\end{cases}
$$

Since $\pi$ intertwines $Q$ with the power two endomorphism $\widehat{Q}$ of $\mathbb{T}$, we obtain (i) and (ii) of the following assertion.

Proposition 3.2. If $T$ is conjugate to $T^{2}$ then

(i) the measure $\widehat{\sigma}$ of the maximal spectral type of $T$ is quasi-invariant under $\widehat{Q}$;

(ii) the spectral multiplicity function $l$ of $T$ satisfies the equation

$$
l(z)=\sum_{\widehat{\sigma}_{z}(v)>0} l(v)
$$

for $\widehat{\sigma}$-a.a. $z$, where $\widehat{\sigma}_{z}$ is the conditional measure of $\widehat{\sigma}$ on the 2-point fiber $\widehat{Q}^{-1} z$ over $Z$;

(iii) if $l$ is bounded then $\widehat{\sigma}$ is singular to Lebesgue measure $\lambda_{\mathbb{T}}$;

(iv) if $T$ has simple spectrum then $\pi$ is one-to-one (on a $\sigma$-conull subset) and hence $\widehat{Q}$ is invertible (on a $\widehat{\sigma}$-conull subset).

Proof. (iii) If there exists a subset $A \subset \mathbb{T}$ with $\lambda_{\mathbb{T}}(A)>0$ such that $\widehat{\sigma}\left\lceil A\right.$ is equivalent to $\lambda_{\mathbb{T}}\lceil A$ then - since $\widehat{\sigma}$ is quasi-invariant under $\widehat{Q}$-we can assume without loss of generality that $\lambda_{\mathbb{T}}(A)=1$. It follows that $\widehat{\sigma}_{z}(v)>0$ for the two points $v \in \widehat{Q}^{-1} z$ at $\lambda_{\mathbb{T}}$-a.a. $z \in \mathbb{T}$. Applying (3-3) several times we come to a contradiction with the boundedness of $l$.

(iv) Since $l(z)=1$ a.e., it follows from (3-2) that $\sigma_{z}$ is supported on a singleton for a.a. $z \in \mathbb{T}$.

REMARK 3.3. If a rank-one transformation $T$ is conjugate to both $T^{2}$ and $T^{3}$ then by Proposition 3.2(i), each measure $\widehat{\sigma}$ of the maximal spectral type for $T$ is quasi-invariant under $\widehat{Q}$ and the power 3 endomorphism of $\mathbb{T}$. Since $T$ has a simple spectrum, we deduce from Proposition 3.2(iii) that $\widehat{\sigma}$ is singular to Lebesgue measure. This provides an answer to a "non-singular" counterpart of Furstenberg's $(\times 2, \times 3)$-problem $[\mathrm{Fu}]$.

4. Compact extensions of transformations conjugate to their squares. Let $\left(T_{h}\right)_{h \in H}$ be a free measure preserving action of $H$ on $(X, \mathfrak{B}, \mu)$. Suppose that the transformation $T_{1}$ is ergodic. Given a compact second countable group $K$, denote by $\mathcal{M}(X, K)$ the group of measurable maps from $X$ to $K$. It is Polish when endowed with the topology of convergence in $\mu$. Let $\alpha: H \ni h \mapsto \alpha_{h} \in \mathcal{M}(X, K)$ be a $T$-cocycle, i.e. $\alpha_{h_{1} h_{2}}=\alpha_{h_{1}} \circ T_{h_{2}} \cdot \alpha_{h_{2}}$ for all $h_{1}, h_{2} \in H$. Then a new $H$-action $T^{\alpha}=\left(T_{h}^{\alpha}\right)_{h \in H}$ is well defined on 
the product space $\left(X \times K, \mu \times \lambda_{K}\right)$ :

$$
T_{h}^{\alpha}(x, k):=\left(T_{h} x, \alpha_{h}(x) k\right) .
$$

It is called the $\alpha$-skew product extension (or an $H$-extension) of $T$. Two cocycles $\alpha$ and $\beta$ of $T$ are cohomologous if there is a map $\phi \in \mathcal{M}(X, K)$ such that $\alpha_{h}=\phi \circ T_{h} \cdot \beta_{h} \cdot \phi^{-1}$ for all $h \in H$. If $T^{\alpha}$ is ergodic then $\alpha$ is called ergodic.

We first investigate a relation between the ergodicity of $\alpha$ and the ergodicity of a single transformation $T_{1}^{\alpha}$.

Proposition 4.1. $\alpha$ is ergodic if and only if there exist a T-cocycle $\beta$ cohomologous to $\alpha$, a closed normal subgroup $K_{0} \subset K$ and a group homomorphism

$$
p: H / \mathbb{Z} \ni h+\mathbb{Z} \mapsto p(h+\mathbb{Z}) \in K / K_{0}
$$

such that the following conditions are satisfied:

(i) $\beta_{1}(x) \in K_{0}$ for a.a. $x \in X$,

(ii) the transformation $(x, k) \mapsto\left(T_{1} x, \beta_{1}(x) k\right)$ of the space $\left(X \times K_{0}, \mu \times\right.$ $\left.\lambda_{K_{0}}\right)$ is ergodic, where $\lambda_{K_{0}}$ stands for the normalized Haar measure on $K_{0}$,

(iii) the range $p(H / \mathbb{Z})$ of $p$ is dense in the quotient group $K / K_{0}$; and hence $K / K_{0}$ is Abelian,

(iv) $\beta_{h}(x) H_{0}=p(h+\mathbb{Z}) H_{0} \in K / K_{0}$ for a.a. $x \in X$ and all $h \in H$.

Proof. We need only verify the "only if" part. The "if" part is trivial. Thus let $\alpha$ be ergodic. By [Zi, Corollary 3.8], there exist a closed subgroup $K_{0} \subset K$ and a cocycle $\beta$ cohomologous to $\alpha$ such that (i) and (ii) are satisfied. Now it follows from [Da1, Lemma 2.1] that for each $h \in H$, there is $p(h) \in N_{K}\left(K_{0}\right)$ such that $\beta_{h}(x) K_{0}=p(h) K_{0}$ for a.a. $x$. Recall that $N_{K}\left(K_{0}\right)$ denotes the normalizer of $K_{0}$ in $K$. The cocycle identity for $\beta$ implies that $p$ is a group homomorphism. Hence $\beta_{h}(X) \subset N_{K}\left(K_{0}\right)$ for all $h \in H$. Since $\alpha$ is ergodic, so is $\beta$. Therefore $N_{K}\left(K_{0}\right)=K$, i.e. $K_{0}$ is normal in $K$. The kernel of $p$ includes $\mathbb{Z}$ by (i). Also, the range of the homomorphism $H \ni h \mapsto p(h) K_{0} \in K / K_{0}$ is dense because $\beta$ is ergodic.

Corollary 4.2. Suppose that the quotient of $K$ by its commutant is totally disconnected (an interesting particular case is when $K$ is finite). Then $\alpha$ is ergodic if and only if the transformation $T_{1}^{\alpha}$ is ergodic.

Proof. Let $\alpha$ be ergodic and $K_{0} \neq K$. Denote by $K^{\prime}$ the commutant of $K$. It follows from Proposition 4.1(iii) that $K_{0} \supset K^{\prime}$. Let $\pi: K / K^{\prime} \rightarrow$ $K / K_{0}$ stand for the canonical projection $k K^{\prime} \mapsto k K_{0}$. Since $K / K^{\prime}$ is totally disconnected, there exists a subgroup $O$ of finite index in $K / K^{\prime}$ such that $\pi(O) \neq K / K_{0}$. Notice that $\pi(O)$ is a subgroup of finite index in $K / K_{0}$. Then it follows from Proposition 4.1(iii) that $p^{-1}(\pi(O))$ is a subgroup of 
the very same finite index in $H / \mathbb{Z}$. We get a contradiction because $H / \mathbb{Z}$ has no proper subgroups of finite index.

We also note that if $K=\mathbb{T}$ then it is easy to construct $T$ and $\alpha$ such that $\alpha$ is ergodic but $T_{1}^{\alpha}$ is not.

Suppose now that $S T_{h} S^{-1}=T_{2 h}$ for all $h \in H$ and a transformation $S \in \operatorname{Aut}_{0}(X, \mu)$. We say that $S$ can be lifted to a conjugacy of $T_{1}^{\alpha}$ and $T_{2}^{\alpha}$ if there exists a transformation $\widetilde{S} \in \operatorname{Aut}_{0}\left(X \times K, \mu \times \lambda_{k}\right)$ with $\widetilde{S} T_{1}^{\alpha} \widetilde{S}^{-1}=T_{2}^{\alpha}$ and $\widetilde{S}(x, k)=(S x, \cdot)$ for a.a. $(x, k) \in X \times K$.

Proposition 4.3. Let $\alpha$ be ergodic. Then $S$ can be lifted to a conjugacy $\widetilde{S}$ of $T_{1}^{\alpha}$ and $T_{2}^{\alpha}$ if and only if there exist a map $a \in \mathcal{M}(X, K)$ and a continuous group automorphism $l: K \rightarrow K$ such that

$$
\alpha_{1} \circ\left(T_{1} S\right) \cdot \alpha_{1} \circ S=a \circ T_{1} \cdot l \circ \alpha_{1} \cdot a^{-1} .
$$

Moreover, the corresponding lift of $S$ is then of the form

$$
\widetilde{S}(x, k)=(S x, l(k) a(x)) \quad \text { for a.a. }(x, k) \in X \times K .
$$

Proof. We first notice that $S$ normalizes the $T$-orbit equivalence relation, i.e. $S\left\{T_{h} x \mid h \in H\right\}=\left\{T_{h} S x \mid h \in H\right\}$ for a.a. $x$. Hence we may apply some results on "lifting" from [Da1] which are of orbital nature. (Observe also that $S$ does not normalize the $T_{1}$-orbit equivalence relation.) It follows from [Da1, Theorem 5.3] that $S$ can be lifted to a conjugacy $\widetilde{S}$ of $T_{1}^{\alpha}$ and $T_{2}^{\alpha}$ if and only if there exists a map $a \in \mathcal{M}(X, K)$ and a continuous group automorphism $l: K \rightarrow K$ such that

$$
\alpha_{2 h} \circ S=a \circ T_{h} \cdot l \circ \alpha_{h} \cdot a^{-1} \quad \text { for all } h \in H
$$

and $\widetilde{S}(x, k)=(S x, l(k) a(x))$. Notice that the system of equations (4-2) is equivalent to a single equation (4-1), which, in turn, is equivalent to $\widetilde{S} T_{1}^{\alpha}=T_{2}^{\alpha} \widetilde{S}$.

It is easy to see that the $T$-cocycles form a closed subset $\mathcal{C}$ of $\mathcal{M}(X, K)^{H}$ endowed with the (Polish) infinite product topology. Applying [Da1, Theorems 5.9 and Theorem 5.8] we deduce the following from Proposition 4.3.

Corollary 4.4.

(i) There is a residual subset of ergodic cocycles $\alpha \in \mathcal{C}$ such that $S$ cannot be lifted to a conjugacy of $T_{1}^{\alpha}$ and $T_{2}^{\alpha}$.

(ii) For each automorphism $l \in$ Aut $K$, there exists an ergodic cocycle $\alpha$ of $T$ such that (4-1) is satisfied, i.e. $S$ can be lifted to a conjugacy of $T_{1}^{\alpha}$ and $T_{2}^{\alpha}$.

Recall that given a dynamical system $(X, \mathfrak{B}, \mu, R)$, every $R$-invariant sub- $\sigma$-algebra is called a factor of $R$. For instance, $\mathfrak{B} \otimes\{\emptyset, K\}$ is a factor of $T_{1}^{\alpha}$. Proposition 4.3 describes a "structure" of the conjugations between 
$T_{1}^{\alpha}$ and $T_{2}^{\alpha}$ that preserve $\mathfrak{B} \otimes\{\emptyset, K\}$. Now we want to specify a condition on a factor of $R$ under which every conjugation of $R$ and $R^{2}$ preserves this factor. For this, we first recall a couple of definitions from the joining theory of dynamical systems [Gl].

Given two transformations $R_{1}, R_{2} \in \operatorname{Aut}_{0}(X, \mu)$, a measure $\nu$ on $X \times X$ is called a joining of $R_{1}$ and $R_{2}$ if $\nu$ is $R_{1} \times R_{2}$-invariant and the coordinate marginals of $\nu$ are both equal to $\mu$. For instance, the product $\mu_{1} \times \mu_{2}$ is a joining of $T_{1}$ and $T_{2}$. Another example is a graph-joining $\mu_{S}$ generated by a transformation conjugating $T_{1}$ with $T_{2}$. It is defined by $\mu_{S}(A \times B):=$ $\mu\left(A \cap S^{-1} B\right), A, B \in \mathfrak{B}$. Of course, $\mu_{S}$ (i.e. the dynamical system $(X \times$ $\left.X, \mu_{S}, R_{1} \times R_{2}\right)$ ) is ergodic. A transformation $R$ is called 2-fold simple if every ergodic joining of $R$ with itself is either the product measure or a graph-joining. For instance, each transformation with pure point spectrum is 2 -fold simple.

Proposition 4.5. Let $T$ be an ergodic transformation and let $\mathfrak{F}$ be a proper factor of $T$. If $T \nmid \mathfrak{F}$ is 2-fold simple and conjugate to its square then every conjugation of $T$ with $T^{2}$ preserves $\mathfrak{F}$ (and hence conjugates $T \nmid \mathfrak{F}$ and $T^{2}(\mathfrak{F})$

Proof. Let $S T S^{-1}=T^{2}$ and let $\mu_{S}$ denote the graph-joining of $T$ and $T^{2}$ generated by $S$. Of course, the sub- $\sigma$-algebra $\mathfrak{F} \otimes \mathfrak{F}$ is a factor of $T \times T^{2}$. Hence $\mu_{S} \uparrow(\mathfrak{F} \otimes \mathfrak{F})$ is an ergodic joining of $T \uparrow \mathfrak{F}$ and $T^{2} \uparrow \mathfrak{F}$. It follows from the assumption on $\mathfrak{F}$ that $\mu_{S} \uparrow(\mathfrak{F} \otimes \mathfrak{F})$ is either the product measure or a graph-joining. It is rather easy to see that the former is impossible. Hence $\mu_{S} \uparrow(\mathfrak{F} \otimes \mathfrak{F})$ is a graph-joining. This implies that $S$ preserves $\mathfrak{F}$.

The next proposition - partly following from Proposition 4.5-extends and refines [Go2, Theorems 3, 4, Corollary 1] and [Go3, Theorems 1, 5].

Proposition 4.6. Let $S T S^{-1}=T^{2}$ with $T$ ergodic. Let $\mathfrak{F}$ denote the Kronecker factor of $T$, i.e. the maximal factor with pure discrete spectrum. Then

(i) the discrete spectrum $\Lambda(T) \subset \mathbb{T}$ of $T$ is invariant under the power two map;

(ii) $\mathfrak{F}$ is invariant under $S$ and hence $T \nmid \mathfrak{F}$ is conjugate to its square;

(iii) $S \nmid \mathfrak{F}$ is ergodic if and only if $T$ is totally ergodic, i.e. $\Lambda(T)$ does not contain non-trivial roots of 1 ;

(iv) if $T$ is totally ergodic but not weakly mixing then $S \nmid \mathfrak{F}$ is Bernoullian.

Proof. (i) Since $T$ and $T^{2}$ are isomorphic, we have $\Lambda(T)=\Lambda\left(T^{2}\right)=$ $\left\{\lambda^{2} \mid \lambda \in \Lambda(T)\right\}$.

(ii) follows from (i) and Proposition 4.5. 
(iii) Since the restriction of $S$ to $\mathfrak{F}$ is isomophic to the "power two" automorphism of the compact dual group $\widehat{\Lambda(T)}$, we deduce (iii) from the standard ergodicity criterion for such maps [CFS, Chapter 3, §3, Theorem 1].

(iv) follows from (iii) and the fact that each ergodic automorphism of a compact group is Bernoullian.

Recall that a transformation $R$ has the weak closure property (WCP) if $C(T)=\mathrm{Cl}_{w}\left(\left\{R^{n} \mid n \in \mathbb{Z}\right\}\right)$, where $\mathrm{Cl}_{w}($.$) denotes the closure in the weak$ topology. It follows that $C(T)$ is a monothetic group. By [Ki1], each rank-one transformation has WCP.

We notice that if $T$ is ergodic then $C(T)$ contains an element of order $q$ if and only if $T$ is a $\mathbb{Z} / q \mathbb{Z}$-extension of another transformation.

Proposition 4.7. Let $T$ have $W C T$ and $S T S^{-1}=T^{2}$. Then the following are satisfied:

(i) (cf. [Go2, Theorem 2]) The map

$$
C(T) \ni R \mapsto S R S^{-1}=R^{2} \in C(T)
$$

is a group isomorphism. Therefore $C(T)=C\left(T^{2}\right)$.

(ii) (cf. [Go2, Proposition 7]) $C(T)$ does not contain non-trivial elements of finite even order.

Proof. (i) Let $V$ be an action of $H$ with $V_{1}=T$. Now it suffices to notice that $C(T)=\mathrm{Cl}_{w}\left(\left\{V_{h} \mid h \in H\right\}\right), S V_{h} S^{-1}=V_{2 h}$ for all $h \in H$ and the homomorphism $H \ni h \mapsto 2 h \in H$ is a bijection of $H$.

(ii) Let $R \in C(T) \backslash\{\mathrm{Id}\}$ be of finite even order. Since $S R S^{-1}=R^{2}$ by (i), the orders of $R$ and $R^{2}$ are the same, a contradiction.

In view of the above, Goodson asks naturally in [Go2] and [Go3]:

(०) for $T$ weakly mixing and satisfying the conditions of Proposition 4.7, can $C(T)$ contain elements of odd order?

We first provide a preliminary "answer" in the class of transformations with pure point spectrum.

EXAMPLE 4.8. Let $T$ be the ergodic transformation with pure point spectrum $\left\{\exp \left(i \theta n / 2^{m}\right) \mid n, m \in \mathbb{Z}\right\}$ for an irrational $\theta$. Then $T$ is conjugate to $T^{2}$. Fix an odd $q$. Let $R$ be a translation by 1 on the cyclic group $\mathbb{Z} / q \mathbb{Z}$. Since the power two automorphism of $\mathbb{Z} / q \mathbb{Z}$ conjugates $R$ with $R^{2}$, the direct product $T \times R$ is conjugate to $T^{2} \times S^{2}$. Moreover, $T \times R$ is ergodic with pure point spectrum. Hence $T \times R$ has WCP. It remains to notice that $\operatorname{Id} \times R$ commutes with $T \times R$.

Since each rank-one transformation has WCP, the following theorem answers (o) affirmatively. 
THEOREM 4.9. Given an odd $q$, there exists a weakly mixing rank-one transformation $T$ conjugate to $T^{2}$ and such that $C(T)$ contains an element of order $q$.

Proof. Let $E$ be the semidirect product $E:=(H \times \mathbb{Z} / q \mathbb{Z}) \rtimes \mathbb{Z}$ with the multiplication

$$
(h, i, n)\left(h^{\prime}, i^{\prime}, n^{\prime}\right):=\left(h+2^{n} h^{\prime}, i+2^{n} i^{\prime}, n+n^{\prime}\right) .
$$

Denote by $\mathcal{A}_{E} \subset \operatorname{Aut}_{0}(X, \mu)^{E}$ the closed subset of $\mu$-preserving actions of $E$ on $(X, \mu)$. As in the proof of Theorem 1.3, it is enough to show that the following two subsets contain free $E$-actions:

$$
\begin{aligned}
& \mathcal{W}_{E}:=\left\{V \in \mathcal{A}_{E} \mid V_{(1,0,0)} \text { is weakly mixing }\right\}, \\
& \mathcal{R}_{E}^{1}:=\left\{V \in \mathcal{A}_{E} \mid V_{(1,0,0)} \text { has rank one }\right\} .
\end{aligned}
$$

Any Bernoullian $E$-action is free and belongs to $\mathcal{W}_{E}$. Since each ergodic transformation with pure point spectrum has rank one [dJ1], it follows from Example 4.7 that $\mathcal{R}_{E}^{1}$ contains a free $E$-action $V$ given by $V_{(1,0,0)}:=T$, $V_{(0,1,0)}:=R$ and $V_{(0,0,1)}:=S$, where $S$ is a transformation conjugating $T \times R$ with $T^{2} \times R^{2}$.

While the above proof is not constructive, we also note that slightly modifying the argument given in the proof of Theorem 2.2, one can construct explicit cut-and-stack examples of $T$ with the properties stated in Theorem 4.9 .

We conclude this section with a "higher rank" analogue of Theorem 1.3.

TheOREM 4.10. For each $n>0$, there exists a weakly mixing transformation $R$ conjugate to its square and such that $n<\operatorname{rk} R<\infty$.

Proof. Let $K_{1}, K_{2}, \ldots$ be a sequence of simple finite groups such that the supremum of the dimensions of the irreducible representations of $K_{n}$ is more than $n$. Let $R$ be a rank-one weakly mixing transformation conjugate to its square. Then there exists an action $V$ of $H$ such that $V_{1}=R$. By Corollary 4.4(ii), for each $n>0$, there exists an ergodic cocycle $\alpha$ of $V$ with values in $K_{n}$ such that (4-1) is satisfied for $l=\mathrm{Id}$. Hence the transformation $V_{1}^{\alpha}$ is conjugate to its square. By Corollary 4.2, the transformation $V_{1}^{\alpha}$ is ergodic. Since $R$ is weakly mixing and $K_{n}$ has no non-trivial homomorphisms to $\mathbb{T}$, it follows that $V_{1}^{\alpha}$ is weakly mixing. Since $R$ is of $\operatorname{rank}$ one, $\operatorname{rk}\left(V_{1}^{\alpha}\right) \leq \# K_{n}$. Let $M_{n}$ denote the essential supremum of the spectral multiplicity function of $V_{1}^{\alpha}$ with respect to the maximal spectral type. Since $M_{n}$ is no less than the maximum of the dimensions of the irreducible representations of $K_{n}$ by [Ro], we obtain $M_{n} \geq n$. It remains to use the fact that $\operatorname{rk}\left(V_{1}^{\alpha}\right) \geq M_{n}[\mathrm{Ch}]$.

Note that $V_{1}^{\alpha}$ does not have WCP because $C\left(V_{1}^{\alpha}\right)$ contains the nonAbelian subgroup $K_{n}$. 
5. Concluding remarks. The following classes of ergodic transformations (plus their direct products) conjugate to their squares are known so far:

$(A)$ the transformations with pure point spectrum whose point spectrum is invariant under the power two homomorphism;

$(B)$ the Bernoullian shifts of infinite entropy;

$(C)$ the time- $(0,1)$ transformations of the $G$-actions induced from $H$ actions;

$(D)$ the time-1 transformations of the horocycle flows on compact connected orientable Riemannian surfaces of constant negative curvature [CFS] or, more generally, on surfaces whose sectional curvatures are negative [Mar] (such transformations are mixing and of infinite rank, some of them are simple);

$(E)$ a class of Gaussian transformations whose spectral measure (not to be confused with the maximal spectral type) is non-atomic and quasi-invariant under the power two homomorphism [Go3] (they are weakly mixing and can have a simple spectrum);

$(F)$ weakly mixing rank-one or, more generally, finite rank transformations constructed in this paper.

Notice that the class $(F)$ is essentially new. It does not intersect any other class: $(E) \cap(F)=\emptyset$ since any Gaussian transformation is of infinite rank $[\mathrm{dR}]$; the fact that $(C) \cap(F)=\emptyset$ was explained in Section 3; the rest is obvious.

It is interesting to find new examples. For instance:

(o) Do there exist interval exchange transformations conjugate to their squares?

(o) Are there mildly mixing non-mixing smooth models for ergodic $T$ conjugate to $T^{2}$ ?

We also conjecture that

( $\widetilde{o})$ the transformation $T_{1}$ constructed in Theorem 2.2 is simple and prime.

\section{References}

[Ag1] O. N. Ageev, The homogeneous spectrum problem in ergodic theory, Invent. Math. 160 (2005), 417-446.

[Ag2] - Spectral rigidity of group actions: applications to the case $\operatorname{gr}\left\langle t, s ; t s=s t^{2}\right\rangle$, Proc. Amer. Math. Soc. 134 (2006), 1331-1338.

[Ch] R. V. Chacon, Approximation and spectral multiplicity, in: Lecture Notes in Math. 160, Springer, 1970, 18-27.

[CFS] I. P. Cornfeld, S. V. Fomin and Ya. G. Sină̌, Ergodic Theory, Grundlehren Math. Wiss. 245, Springer, New York, 1982. 
[Da1] A. I. Danilenko, Comparison of cocycles of measured equivalence relations and lifting problems, Ergodic Theory Dynam. Systems 18 (1998), 125-151.

[Da2] -, On cocycles with values in group extensions. Generic results, Mat. Fizika Analiz Geom. 7 (2000), 153-171.

[Da3] -, Funny rank one weak mixing for nonsingular Abelian actions, Israel J. Math. 121 (2001), 29-54.

[Da4] - Infinite rank one actions and nonsingular Chacon transformations, Illinois J. Math. 48 (2004), 769-786.

[Da5] - Explicit solution of Rokhlin's problem on homogeneous spectrum and applications, Ergodic Theory Dynam. Systems 26 (2006), 1467-1490.

[Da6] -, On simplicity concepts for ergodic actions, J. Anal. Math. 102 (2007), 77-118.

[Da7] - , $(C, F)$-actions in ergodic theory, in: Geometry and Dynamics of Groups and Spaces, Progr. Math. 265, Birkhäuser, 2007, 325-351.

[DdJ] A. I. Danilenko and A. del Junco, Cut-and-stack simple weakly mixing map with countably many prime factors, Proc. Amer. Math. Soc. 136 (2008), 2463-2472.

[DaS] A. I. Danilenko and C. E. Silva, Multiple and polynomial recurrence for abelian actions in infinite measure, J. London Math. Soc. 69 (2004), 183-200.

[D-S] A. N. Dooley, V. Ya. Golodets, D. J. Rudolph and S. D. Sinel'shchikov, NonBernoulli systems with completely positive entropy, Ergodic Theory Dynam. Systems 28 (2008), 87-124.

[FoW] M. Foreman and B. Weiss, An anti-classification theorem for ergodic measure preserving transformations, J. Eur. Math. Soc. 6 (2004), 277-292.

[Fu] H. Furstenberg, Disjointness in ergodic theory, minimal sets, and a problem in Diophantine approximation, Math. Systems Theory 1 (1967), 1-49.

[Gl] E. Glasner, Ergodic Theory via Joinings, Math. Surveys Monogr. 101, Amer. Math. Soc., Providence, RI, 2003.

[GlK] E. Glasner and J. L. King, A zero-one law for dynamical properties, in: Topological Dynamics and Applications (Minneapolis, MN, 1995), Contemp. Math. 215, Amer. Math. Soc., Providence, RI, 1998, 231-242.

[Go1] G. R. Goodson, A survey of recent results in the spectral theory of ergodic dynamical systems, J. Dynam. Control Systems 5 (1999), 173-226.

[Go2] —, Ergodic dynamical systems conjugate to their composition squares, Acta Math. Univ. Comenian. (N.S.) 71 (2002), 201-210.

[Go3] - Spectral properties of ergodic dynamical systems conjugate to their composition squares, Colloq. Math. 107 (2007), 99-118.

[Ha] P. R. Halmos, Lectures on Ergodic Theory, Chelsea, New York, 1960.

[dJ1] A. del Junco, Transformations with discrete spectrum are stacking transformations, Canad. J. Math. 28 (1976), 836-839.

[dJ2] —, Disjointness of measure-preserving transformations, minimal self-joinings and category, in: Ergodic Theory and Dynamical Systems, I (College Park, MD, 1979-80), Progr. Math. 10, Birkhäuser, Boston, MA, 1981, 81-89.

[dJ3] - A simple map with no prime factors, Israel J. Math. 104 (1998), 301-320.

[KaS] A. Katok and A. Stepin, Approximations in ergodic theory, Uspekhi Mat. Nauk 22 (1967), no. 5, 81-106 (in Russian).

[Ki1] J. King, The commutant is the weak closure of the powers, for rank-1 transformations, Ergodic Theory Dynam. Systems 6 (1986), 363-384.

[Ki2] - , The generic transformation has roots of all orders, Colloq. Math. 84/85 (2000), 521-547.

[Ma] B. Madore, Rank-one group actions with simple mixing $\mathbb{Z}$-subactions, New York J. Math. 10 (2004), 175-194. 
[Mar] B. Marcus, Unique ergodicity of the horocycle flow: variable negative curvature case, Israel J. Math. 21 (1975), 133-144.

[Ro] E. A. Robinson, Jr., Nonabelian extensions have nonsimple spectrum, Compos. Math. 65 (1988), 155-170.

[dR] T. de la Rue, Rang des systèmes dynamiques gaussiens, Israel J. Math. 104 (1998), 261-283.

[dRdS] T. de la Rue et J. de Sam Lazaro, Une transformation générique peut être insérée dans un flot, Ann. Inst. H. Poincaré Probab. Statist. 39 (2003), 121-134.

[Ry] V. V. Ryzhikov, Self-joinings of rank-one actions and applications, Sém. Congr. 19, Soc. Math. France, 2008.

[Zi] R. Zimmer, Extensions of ergodic group actions, Illinois J. Math. 20 (1976), 373-409.

Max Planck Institute for Mathematics

Vivatsgasse 7

Bonn, 53111, Germany

Permanent address:

Institute for Low Temperature Physics \& Engineering

of Ukrainian National Academy of Sciences

47 Lenin Ave.

Kharkov, 61164, Ukraine

E-mail: danilenko@ilt.kharkov.ua

Received December 11, 2006

Revised version March 29, 2008 